\section{Pricktest: Welche Ablesezeit ist die beste?}

\author{
Der Pricktest ist für Allergologen ein alt bekanntes und bewährtes \\ Verfahren. Weniger bekannt war bisher, wann eigentlich der beste \\ Zeitpunkt ist, das Ergebnis des Tests abzulesen. Diese Frage wollten \\ US-amerikanische Wissenschaftler nun klären.
}

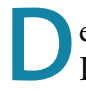
er Pricktest ist als Routinemethode, IgE-vermittelte Sensibilisierungen zu diagnostizieren, anerkannt. Testquaddel und der diese umgebende Erythemhof werden routinemäßig nach 15 bis 20 Minuten abgelesen. $\mathrm{Ob}$ eine frühere oder spätere Ablesung den diagnostischen Aussagewert beeinträchtigt, sollte untersucht werden.

Hierzu wurden bei 57 Patienten verschiedenen Alters und Geschlechts Pricktests mit unterschiedlichen Allergenen durchgeführt und nach 10, 20, 30 und $40 \mathrm{~min}$ die Testquaddel und das umgebende Erythem vermessen. Patienten mit Dermografismus waren ausgeschlossen. Quaddeln und Erytheme, die 20 min nach Testbeginn auftraten,

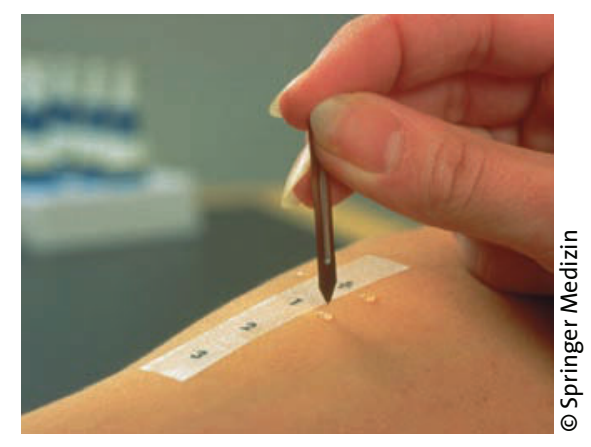
20 Minuten nach dem Prick.

wurden in ihrer Größe mit denen nach 10, 30 und 40 min verglichen.

Die statistische Auswertung ergab eine sehr enge Korrelation der Flächen-

\section{Die optimalste Ablesezeit ist 15 bis}

größen zwischen 20- und 30-minütiger Ablesung sowie eine deutlich schwächere zwischen 10 und $40 \mathrm{~min}$. Eine Ablesung nach weniger als 10 und mehr als $40 \mathrm{~min}$ wurde zunehmend ungenauer.

Fazit: Beim Pricktest sollte die übliche und die optimale Ablesung gewährleistende Ablesezeit von 15 bis 20 min nach Durchführung des Pricks eingehalten werden. Verhindern jedoch äußere Umstände die zeitlich korrekte Ablesung, kann für einen Zeitraum von 10 bis 40 min nach dem Prick noch ohne wesentlichen Verlust des diagnostischen Aussagewertes abgelesen werden. Ablesezeiten unter $10 \mathrm{~min}$ und über $40 \mathrm{~min}$ bedeuten dagegen einen erheblichen Qualitätsverlust.

Dr. Dieter Bruchhausen

Seibert SM et al. Reliability of skin test results when read at different time points. Allergy Asthma Proc 2011; 32: 203-5

Die Spiegel der regulatorischen Th1-Zytokine stiegen sowohl unter SLIT als auch unter SCIT. Eine $\mathrm{Zu}$ nahme der antigenspezifischen IgG4Konzentration war hingegen nur bei Beteiligung der SCIT und nicht bei alleiniger SLIT zu beobachten. Die klinischen Beschwerden korrelierten mit den Pegeln an spezifischem IgG4, IFN- $\gamma$ und TGF- $\beta$.

Fazit: SCIT in der Aufbauphase, SLIT in der Erhaltungsphase: So lautet das Rezept für die pädiatrische spezifische Immuntherapie nach den Ergebnissen der vorliegenden Studie. Dieses Regime soll die schnelle therapeutische Wirkung der SCIT mit dem Sicherheitsprofil der SLIT vereinen - und die bei Kindern wenig beliebten Injektionen nach viermonatiger Aufbautherapie vermeiden helfen.

Dr. Robert Bublak

Keles $\mathbf{S}$ et al. A novel approach in allergen-specific immunotherapy: Combination of sublingual and subcutaneous routes. J All Clin Immunol 2011; 128: 808-15 